\title{
On hyperbolic comets
}

\author{
A. S. Guliyev and A. S. Dadashov \\ Nassriddin Tussi Astrophysical Observatory of National Academy of Sciences of Azerbaidjan \\ Republic, ShAO. \\ Mammadaliyev settlement, Pirgulu, Shamaky, AZ5613, Azerbaijan Republic, \\ email: ayyub54@yahoo.com
}

\begin{abstract}
Preliminary results of an investigation on the problem of hyperbolic comets are presented and discussed.
\end{abstract}

Keywords. comets: general, hyperbolic orbit, inter-orbital distance, perihelion concentration

\section{Introduction}

According to the existing practice, comets are considered to be hyperbolic ones if their "original" $1 / a$ (hereinafter $1 / a$ ) are negative. Number of such comets, according to the catalogue by Marsden and Williams (2008), is equal to 34. Negative 1/a for three other long-period comets were calculated later. So, the present work contains results of the analysis of 37 long-period comets.

As is known, there are various works on hyperbolic comets (hereinafter $H C s$ ) in the scientific literature. Existing widely accepted and less popular versions regarding to the origin of $\mathrm{HCs}$ could be classified as follows:

1. Hyperbolic excess of eccentricity is formed as a result of physical processes occurring on comet nucleus;

2. Hyperbolic excess of eccentricities could be results of errors in determination of orbits, or "original" ones;

3. HCs might have an interstellar origin;

4. Hyperbolic excesses of eccentricities are result of unaccounted factors.

Authors' methodological approach in study of $H C s$ is specific. We consider $H C s$ not only as set of individual long-period comets, but as a whole system. This system can have specific characteristics, which are not inherent for all cometary system.

During the preliminary analysis we have found following specific characteristics of $H C$ :

1. Values of $1 / a$ of $H C s$ vary from -1 up to $-772\left(1\right.$ unit $=10^{-6}$ a.u. $\left.{ }^{-1}\right)$; they are distributed not chaotically and exponentially (fig. 1). Thus distributions of $1 / a$ of $H C$ for class 1 and 2 (Marsden and Williams, 2008) are slightly different.

2 . The ratio of direct and retrograde orbits is $16: 21 ; 18$ from $37 \mathrm{HC}$ 's perihelion are concentrated in two narrow and opposite intervals of longitudes, namely in $49^{0}-102^{0}$ and $248^{0}-292^{0}$;

3. Values of absolute brightness for $20 \mathrm{HCs}$ are determined: they are distributed in the interval from $5^{m} .2$ to $12^{m} .4$. Hence, comets are not unusual in sense of brightness. There is an inverse correlation between parameters $H_{10}$ and $1 / a$ for 20 considered comets (correlation coefficient is -0.45 ).

4. Noticeable concentration of $H C$ 's perihelion close to the solar apex, solar equator's and galaxy plane does not exist.

5. The fraction of $H C s$ gradually grows in the total list of comets. It excludes the version that they are results of mistakes of calculations; 


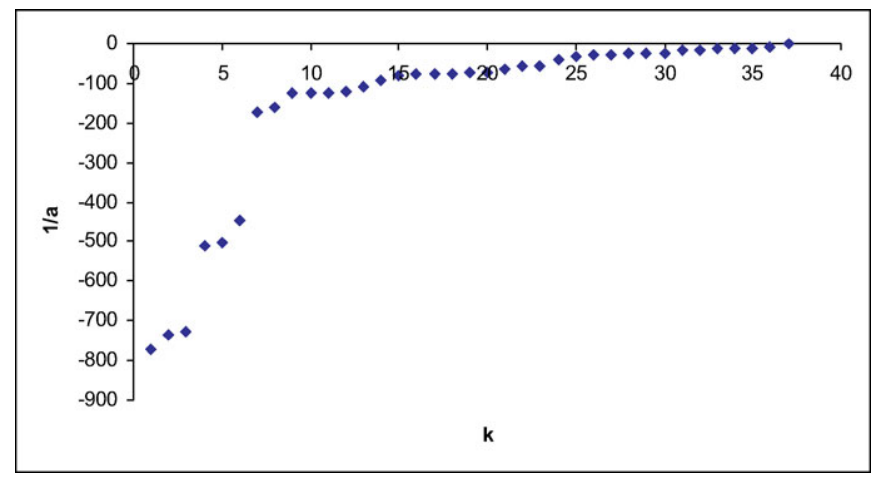

Figure 1. Increasing order of values of $1 / a$ of hyperbolic comets.

6. HCs practically do not differ from other long-periodic comets on parameters $L, q$ and $i$.

It is necessary note that absence of distinction of $H C$ from general ensemble on parameters $q$ and $H_{10}$ (probably absence of connection with solar equator also) creates difficulties for the version about only non-gravitational nature of hyperbolic excess of $H C s$ velocity. At the same time some features noted in items 1, 4 and 5 creates difficulties for the assumption of an interstellar origin of such comets.

Now we will state some results of our analysis and calculations on $\mathrm{HCs}$ :

1. Some previous researches (Guliyev, 1999,2007) showed that perihelion of long-period comets, except for some groups with small $q$, are concentrated near the plane with parameters $I=86^{0}$ and $\Omega=273^{0}$. In addition it was found, that remote nodes of long-period comets orbits concerning this plane have overpopulation in the interval $250-400$ a.u. Some excess of aphelion number on this interval exists also (Guliyev 2008). One of authors in his relevant works has advanced hypothesis that in this plane and on the distances $250-400$ a.u. there might be very big kuiper body. This body could be a source of generation of comet nucleus from transneptunian zones in sphere of visibility. Developing this hypothesis, we can assume that this hypothetical planet body might be reason of acceleration of heliocentric velocity of some $\mathrm{HCs}$. Our analogical calculations show that perihelion of $\mathrm{HCs}$ have very noticeable concentration near the plane

$$
I=87^{0} ; \quad \Omega=274^{0} .
$$

Perihelion of $15 \mathrm{HC}$ are located in $10^{0}$ latitude of this plane (fig.2). This plane is very close to the previous one. However, degree of concentration in the case of $H C s$ is sharper.

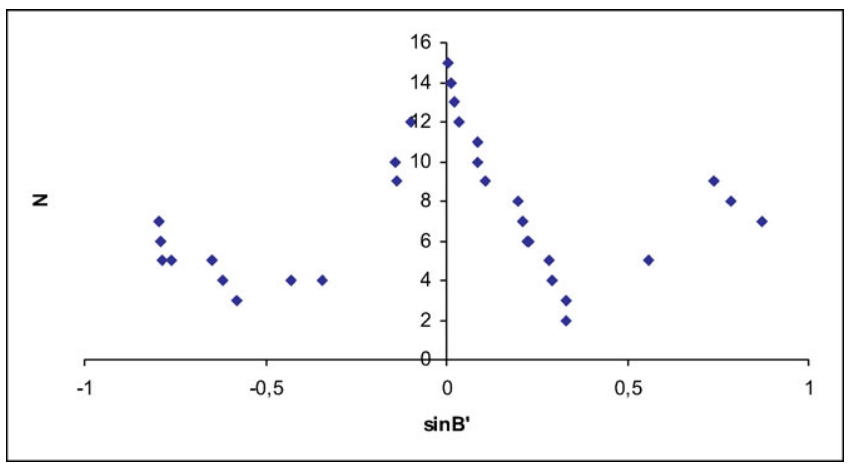

Figure 2. Distribution of hyperbolic comets' perihelion on intervals $\sin B^{\prime}$ (lengths of interlacing intervals are equal to 0.33 ) concerning the plane (1.1). 
2. The ratio of direct and retrograde movements concerning the plane (1.1) is $23: 14$ and it differs from the elliptical one.

3. Authors have analyzed the data $\left(1 / a, \cos I^{\prime}\right.$ and $\left.q\right)$ of 37 comets, entering the Tisseran's criterion for checking an above-mentioned idea. Our calculations showed that there was some multiple correlation dependence between them. The following empirical formula has been found

$$
1 / a=(-155 \pm 66) \cos I^{\prime}-(40 \pm 15) q
$$

( $I^{\prime}$ is inclination of comets orbit concerning accordingly plane hereinafter). Level of determinacy of this expression and its confidentiality probability are 0.26 and 0.99 accordingly. For the class of comets $1 A$ and $1 B$ (Marsden and Williams, 2008) empirical expressions become more certain. It means that 15 comets, or some part of them, can have the certain community connected with the plane (1.1).

4. Authors tried to check up the hypothesis that perihelion of $H C$ are concentrated near several planes. Indeed, if we will exclude 15 comets from list of $H C s$, other 22 ones will tend to have concentration of perihelion near the plane with parameters

$$
I=18.6^{0} ; \quad \Omega=287.2^{0}
$$

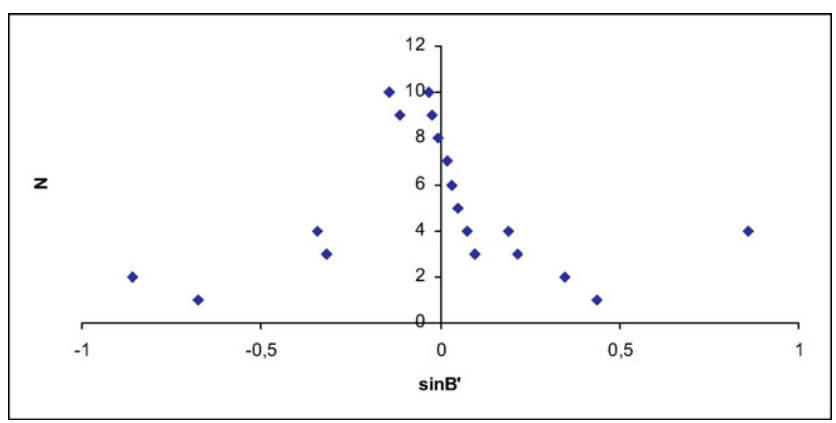

Figure 3. Distribution of hyperbolic comets' perihelion on intervals $\sin B^{\prime}$ (lengths of interlacing intervals are equally to 0.25$)$ regarding the plane $(2.2)$.

Perihelion of $10 \mathrm{HC}$ are located in $10^{\circ}$ latitude of this plane (fig.3). Multiple correlation dependence between parameters $1 / a, q$ and $\cos I$ in this system exists also. The following empirical formula for $22 \mathrm{HC}$ has been obtained

$$
q=-(0.0053 \pm 0.0021) / a-(1.473 \pm 0.744) \cos I^{\prime} .
$$

The ratio of direct and retrograde movements concerning this plane is $7: 15$. We tried to find distinctive features of this plane. After relevant calculations we have found that remote nodes of long-period comets regarding this plane have some excess in the interval 122-137 A.U. We have used the method of testing used in (Guliyev, 2007) for a basing of this prospective excess. Particularly keeping interval $122-137$ A.U., parameters $\Omega\left(0^{0}, 30^{0}, 60^{0} \ldots 330^{\circ}\right)$ and $i\left(0^{0}, 9^{0} .49,19^{0} .47,30^{0}, 41^{0} .81,56^{0} .44\right.$ and $\left.90^{\circ}\right)$ were varied in such way, that poles of corresponding 67 planes were equally removed from each other. Number of comet nodes in fixed interval was found for each plane in the next step of calculations. Consequently we had conducted comparisons using methods of statistics 
and obtained next values:

$$
N=21 ; \quad n=7.73 ; \quad \sigma=2.57 ; \quad t=5.2 ; \quad \alpha=0.999,
$$

where $N, n, \sigma, t$ and $\alpha$ are number of nodes regarding (2.2), midrange value on other planes, normalized difference $(t=(N-n) / \sigma)$, rms deviation and confidential probability of $t$ accordingly. These figures give some bases to assume, that in a plane (2.2) on distances $122--137$ A.U. might be a latent source of comets. The same source could cause increase in heliocentric velocity of some long-period comets.

5. We have calculated minimal inter-orbital distances of $H C s$ from 4 dwarf planets also. 13 of them have distances less than 2 a.u. (Pluto-5, Makemake-1, Eris-6 and Haumea-1). We have found that 8 other comets have such inter-orbital distances from four bright TNO (Orcus-4, Varuna-1, Ixion -1, Quaoar-1). It means that possible influence of these and other TNOs in discussion of the question on hyperbolic comets should be taken into account.

\section{Conclusions}

1. Rate of growth $\mathrm{HCs}$ exceeds rate of growth of other long-period comets. It means at least that system of such comets really exists.

2. Absence of concentration of HCs perihelion near the Galaxy's plane, solar equator plane and the solar apex, as well as patterns in distribution of $1 /$ a excludes the version of interstellar origin of $\mathrm{HCs}$.

3. Absence of distinction of $H C s$ from general ensemble on parameters $q$ and $H_{10}$ creates some difficulties for the version about only non-gravitational nature of hyperbolic excess of $H C s$ velocity.

4. Perihelion of 25 from $37 \mathrm{HCs}$ are concentrated near two planes: $\left(\Omega=273^{0} ; I=86^{0}\right)$ and; $\left(\Omega=287^{0} ; I=19^{0}\right)$. Some $H C s$ could obtain an excess of velocity from hypothetical planet bodies moving in these planes.

5. Hyperbolic comets, by their removed units and inter-orbital distances, could have approaching to dwarf planets and some big kuiper bodies in the past.

\section{References}

Marsden, B. G. \& Williams, G. V. 2005, Catalogue of Cometary Orbits, 16-th edition. (SAO, Cambridge.), 207P

Guliyev, A. S. 2007, Astronomical letters, 33, 8, pp. 562-570

Guliyev, A. S. 1999, Kinematics and physics of celestial bodies, 15, 1, pp. 85-92

Guliyev, A. S. 2008, Azerbaijani Astronomical Journal, 2, 1-2, pp. 5-9 